\title{
Tubingen Zebrafish
}

National Cancer Institute

\section{Source}

National Cancer Institute. Tubingen Zebrafish. NCI Thesaurus. Code C79982.

The wild-type short fin zebrafish line used by the Sanger institute for the Zebrafish sequencing project. The line was cleaned up to remove embryonic lethal mutations from the background before being used for mutagenesis and sequencing. 